\title{
Local Chemical Environments and the Phonon Partial Densities of States of ${ }^{57} \mathrm{Fe}$ in ${ }^{57} \mathrm{Fe}_{3} \mathrm{Al}$
}

\author{
B. Fultz, ${ }^{1}$ T. A. Stephens, ${ }^{1}$ W. Sturhahn, ${ }^{2}$ T. S. Toellner, ${ }^{2}$ and E. E. Alp ${ }^{2}$ \\ ${ }^{1}$ Division of Engineering and Applied Science, 138-78, California Institute of Technology, Pasadena, California 91125 \\ ${ }^{2}$ Advanced Photon Source, Argonne National Laboratory, Argonne, Illinois 60439
}

(Received 15 October 1997)

\begin{abstract}
Inelastic nuclear resonant scattering spectra were measured on alloys of $\mathrm{Fe}_{3} \mathrm{Al}$ that were chemically disordered, partially ordered, and $\mathrm{DO}_{3}$ ordered. The features in the phonon partial density of states of ${ }^{57} \mathrm{Fe}$ were found to change systematically with chemical short-range order in the alloy. Changes in the phonon partial density of states were modeled successfully by assigning vibrational spectra to ${ }^{57} \mathrm{Fe}$ atoms in different first-nearest-neighbor chemical environments. [S0031-9007(98)05811-6]
\end{abstract}

PACS numbers: $76.80 .+\mathrm{y}, 63.20 .-\mathrm{e}, 81.30 . \mathrm{Bx}$

Phase diagrams of materials have been subjects of extensive experimental and theoretical research. In recent years, much of the free energies of solid phases have been calculated by elegant combinations of methods for calculating the electronic energy with the local density approximation and methods using cluster approximations for calculating the configurational entropy [1]. The change in vibrational entropy during a solid state phase transition is often thermodynamically important, however. There is now a growing effort to understand the reasons for differences in vibrational entropy between different states of materials [2-8]. Knowledge of the phonon density of states is central to any understanding of vibrational entropy, at least in the harmonic approximation. Phonons are delocalized excitations on the crystal lattice, so it is not obvious how to include vibrational entropy into methods for phase diagram calculations that are based on the energetic and statistical properties of local clusters of atoms.

It would be particularly convenient if changes in the phonon density of states (DOS) could be assigned to changes in local atomic arrangements. The Einstein model for vibrational dynamics is easily included in mean-field calculations of free energy [4]; and vibrational effective cluster interactions, based on local atomic configurations, have been developed recently [6]. There is some evidence that the vibrational contribution to alloy thermodynamics may depend on short-range features of atomic arrangements. The ranges of interatomic force constants, obtained from the shapes of phonon dispersion curves, are typically not much larger than interatomic distances. Although phonons have many wavelengths, most of the phonons in a solid have high frequencies and short wavelengths. Nevertheless, there have been no experimental tests of how the phonon DOS depends on local atomic configurations of alloys. The present investigation was undertaken to measure the phonon partial density of states of ${ }^{57} \mathrm{Fe}$ atoms in bcc alloys of ${ }^{57} \mathrm{Fe}_{3} \mathrm{Al}$. The alloys had well-characterized differences in chemical short-range order (SRO), so it was possible to correlate the phonon partial DOS to the local chemical environment of the ${ }^{57} \mathrm{Fe}$ atoms.

Previous calorimetric measurements [7] found a difference of $(0.1 \pm 0.03) k_{B} /$ atom in the vibrational entropies of chemically ordered and disordered $\mathrm{Fe}_{3} \mathrm{Al}$, with the disordered alloy having the larger vibrational entropy. This difference in vibrational entropy could be understood from the phonon density of states [7] calculated with the interatomic force constants derived from fits to experimental phonon dispersion curves $[9,10]$. The agreement was only qualitative, however, with the phonon DOS curves providing a difference in vibrational entropy nearly a factor of 2 too large. This discrepancy likely originated with the use of the virtual crystal approximation for the analysis of the coherent inelastic neutron scattering data from chemically disordered $\mathrm{Fe}_{3} \mathrm{Al}$. The virtual crystal approximation does not allow for high frequency vibrations in disordered alloys at the frequencies of optical modes in the ordered alloys. It, therefore, may overestimate the change in phonon DOS upon chemical ordering. High frequency vibrational states in a disordered alloy probably do not exhibit long-range translational symmetry, and are best measured with methods employing incoherent scattering. This motivated the present study using the new technique of inelastic nuclear resonant scattering [11,12].

The measurements were performed at the 3-ID undulator beam line of the Advanced Photon Source. A premonochromater consisting of water-cooled diamond (111) crystals in a nondispersive setting produced a beam of $14.413 \mathrm{keV}$ synchrotron radiation with an energy bandwidth of $1.2 \mathrm{eV}$. Further monochromatization to an $875 \mu \mathrm{eV}$ bandwidth was provided by a high-resolution monochromator [13] comprising two asymmetically cut silicon (975) crystals in a dispersive geometry. The photon flux incident on the foil sample within this bandwidth was $6 \times 10^{8} \mathrm{~Hz}$. The energy alignment of the highresolution monochromater was tuned around the nuclear resonance in steps of $300 \mu \mathrm{eV}$. An avalanche photodiode with an active area of $2 \mathrm{~cm}^{2}$ was mounted $4 \mathrm{~mm}$ above the specimen and used for the detection of time-delayed $K$-shell internal conversion $\mathrm{x}$ rays of $6.4 \mathrm{keV}$. All measurements were performed at room temperature.

Measurements were performed on three foils of ${ }^{57} \mathrm{Fe}_{3} \mathrm{Al}$ of $3 \mu \mathrm{m}$ thickness. They were prepared by arc melting a $50 \mathrm{mg}$ ingot under an argon atmosphere, followed by piston-anvil quenching into a thin foil and cold rolling. A 
JEOL Superprobe 733 electron microprobe was used to check for chemical heterogeneities, and to determine that the chemical composition of the foil was Fe-26.0 at. \% Al. One specimen was used directly in this state, and is denoted as the "disordered" sample. A second foil of ${ }^{57} \mathrm{Fe}_{3} \mathrm{Al}$ was annealed at $473 \mathrm{~K}$ for $1 \mathrm{~h}$ to induce partial chemical order, and is denoted as the "partially ordered" sample. The third foil of ${ }^{57} \mathrm{Fe}_{3} \mathrm{Al}$ was annealed at $773 \mathrm{~K}$ for 6 days, followed by $723 \mathrm{~K}$ for 40 days, and is denoted as the "ordered" sample. The states of chemical longrange order (LRO) of the three samples of ${ }^{57} \mathrm{Fe}_{3} \mathrm{Al}$ were determined by $\mathrm{x}$-ray diffractometry and by conversion electron Mössbauer spectrometry. Some x-ray diffraction patterns are presented in Fig. 1. Only the ordered sample shows any $\mathrm{DO}_{3}$ LRO, as evidenced by intense $\left(\frac{1}{2} \frac{1}{2} \frac{1}{2}\right)$ and (100) $x$-ray superlattice diffractions. The diffraction pattern from the partially ordered sample may show some broad intensity from about $28^{\circ}-37^{\circ}$ that could be diffuse scattering originating with chemical SRO.

Conversion electron Mössbauer spectrometry was used to measure the chemical short-range order in the samples (Fig. 2). A perfect state of $D 0_{3}$ chemical order would have $\mathrm{Fe}$ atoms with only zero or four $\mathrm{Al}$ neighbors (the $4[\mathrm{~b}]$ and 8 [c] sites in Wyckoff notation) in a 1:2 ratio. The excess of $\mathrm{Fe}$ atoms with three $\mathrm{Al}$ neighbors in our ordered sample indicates a LRO parameter between 0.7 and 0.8 (where perfect $\mathrm{DO}_{3}$ order would be 1.0). The disordered sample shows a much broader distribution of local chemical environments, peaking at two first-nearest-neighbor (1nn) Al atoms as expected for a random solid solution. For the disordered alloy, the probability of finding an Fe atom with the number $n$ of $1 \mathrm{nn} \mathrm{Al}$ atoms follows approximately the binomial distribution [14], although there was a small excess of $\mathrm{Fe}$ atoms with four $\mathrm{Al}$ neighbors. The partially ordered sample shows a hyperfine magnetic field (HMF) distribution that is intermediate between the ordered and disordered alloy.

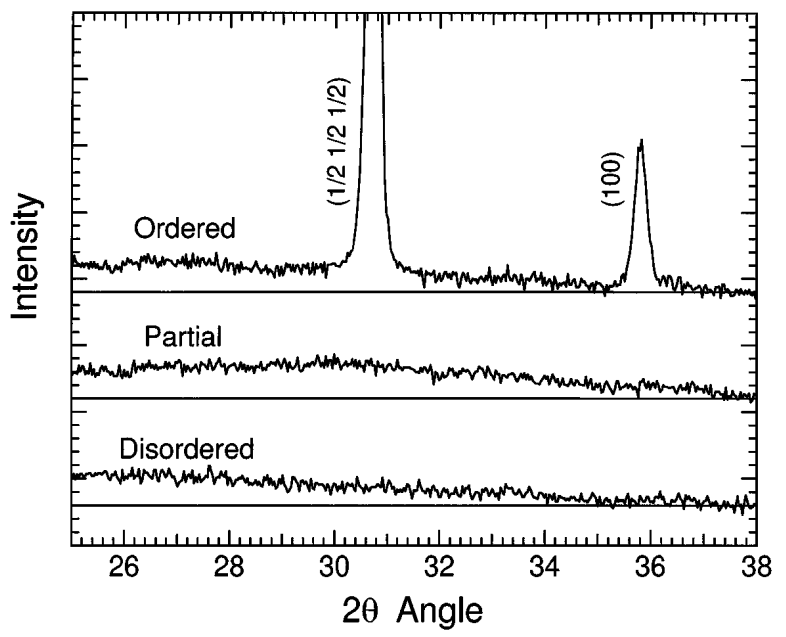

FIG. 1. X-ray diffraction patterns (Co $K \alpha$ radiation, DebyeScherrer optics) from the three specimens of ${ }^{57} \mathrm{Fe}_{3} \mathrm{Al}$ scaled for equal intensities of the (110) fundamental diffraction at $52^{\circ}$.
Inelastic nuclear resonant scattering measurements can provide the phonon partial DOS of ${ }^{57} \mathrm{Fe}$ atoms, $g_{\mathrm{Fe}}(\varepsilon)$, defined as

$$
g_{\mathrm{Fe}}(\varepsilon)=\sum_{\mathbf{d}} \sum_{\gamma} \sum_{\mathbf{q}}\left|\sigma_{d}^{\gamma}(\mathbf{q})\right|^{2} \delta\left(\varepsilon-\varepsilon_{\gamma}(\mathbf{q})\right),
$$

where $\sigma_{d}^{\gamma}(\mathbf{q})$ is the polarization vector for the $\mathrm{Fe}$ atom at the site, d, of the phonon in the branch, $\gamma$, with wave vector, $\mathbf{q}$. In the $\mathrm{DO}_{3}$ ordered structure, the phonon partial DOS involves d vectors for only the 4[b] and $8[c]$ crystallographic sites. Previous study of the phonon partial DOS for $\mathrm{Al}$ and $\mathrm{Fe}$ atoms showed that in optical modes around $43 \mathrm{meV}$, the $\mathrm{Al}$ atoms have much larger vibrational amplitudes than do Fe atoms [7]. The 8[c] $\mathrm{Fe}$ sites form a cage around the $\mathrm{Al}$ atoms, however. For these high energy phonons the polarization vector $\sigma_{8[\mathrm{c}]}^{\gamma}(\mathbf{q})$ is non-negligible.

Inelastic nuclear resonant scattering spectra are presented in Fig. 3. The Mössbauer elastic peak at $E=$ $0 \mathrm{meV}$ is surrounded by the inelastic contribution from nuclear resonant photon absorptions accompanied by phonon excitation (right), and phonon absorption (left). The vibrational modes around $43 \mathrm{meV}$ are present in the disordered alloy, but show a distinct growth in intensity with the chemical order in the alloy. The nonzero intensity at energies above $50 \mathrm{meV}$ is primarily multiphonon scattering $[16,17]$. We corrected for the multiphonon scattering by an iterative procedure [18] and a Fourier-logarithm deconvolution method [11] described previously. These spectra were converted into phonon partial DOS curves for ${ }^{57} \mathrm{Fe}$, and results are shown in the middle of Fig. 4.

At the top of Fig. 4 are phonon partial DOS curves for $\mathrm{Fe}$, calculated with the Born-von Kármán model using interatomic force constants from inelastic neutron scattering $[7,9,10]$ and convoluted with a Gaussian function of $850 \mu \mathrm{eV}$ full width at half maximum to account approximately for the resolution of the monochromator. The partial DOS curve for the disordered alloy was calculated with force constants obtained from bcc dispersion

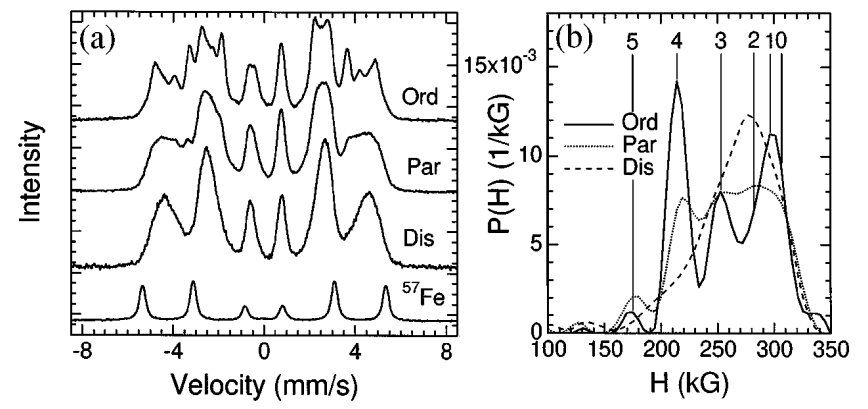

FIG. 2. (a) Conversion electron Mössbauer spectra from the specimen of bcc ${ }^{57} \mathrm{Fe}$ and the three specimens of $\mathrm{DO}_{3}$-ordered ${ }^{57} \mathrm{Fe}_{3} \mathrm{Al}$. (b) $\mathrm{HMF}$ distributions of the ${ }^{57} \mathrm{Fe}_{3} \mathrm{Al}$ specimens extracted by the method of [15]. Peaks in the HMF distribution are labeled with numbers indicating the different numbers of 1nn Al neighbors about the ${ }^{57} \mathrm{Fe}$ atom. 


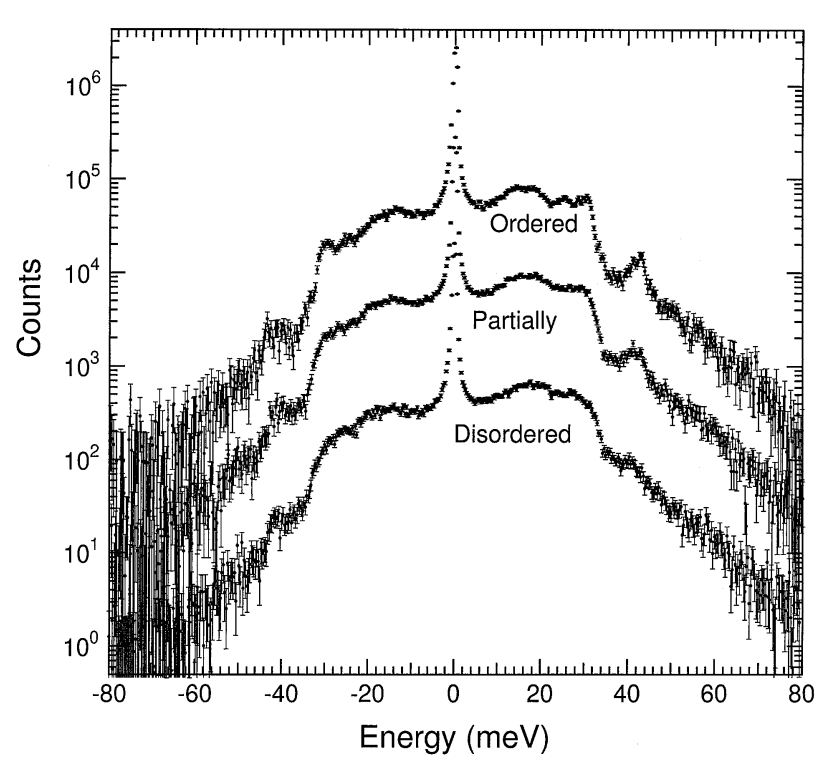

FIG. 3. Conversion x-ray intensity versus photon energy from the disordered, partially ordered, and ordered alloys near the elastic (Mössbauer) resonance set at $0 \mathrm{meV}$. Curves for the partially ordered and ordered samples are shifted vertically by factors of 10 and 100, respectively.

curves [10], which implicitly assumes that the alloy contains one species of atom with the average mass of Fe and Al. This virtual crystal approximation is intrinsic to the analysis of coherent inelastic neutron scattering experiments on disordered alloys when single phonon frequencies are used to parametrize the neutron spectra measured at constant $Q$. The virtual crystal calculation has particularly poor agreement with the experimental data from the disordered alloy for energies above $34 \mathrm{meV}$ in the range of the optical modes of the ordered alloy. On the other hand, for $\mathrm{DO}_{3}$-ordered $\mathrm{Fe}_{3} \mathrm{Al}$ we find good agreement between the calculated partial DOS and our experimental data (where the calculated curve is the sum of two partial DOS curves for the $4[\mathrm{~b}]$ and $8[\mathrm{c}]$ crystallographic sites, shown as dashed lines at the top of Fig. 4). The only distinct discrepancy occurs around $26 \mathrm{meV}$, although this discrepancy vanishes if the calculated curves are broadened by about $2 \mathrm{meV}$. The experimental data also show intensity in the energy gap around $34-38 \mathrm{meV}$ owing to some disorder in the ordered alloy. The intensity in this energy gap becomes larger for the partially ordered and disordered alloys. With increasing disorder there is a decrease in intensity of the DOS at optical mode energies, and these modes also decrease in energy.

The results of Fig. 4 are useful for determining the spatial range of chemical order that affects the phonon DOS. Two of the samples have chemical SRO, but only one has LRO. From the systematic changes in the phonon partial DOS with chemical SRO, it is clear that the ${ }^{57} \mathrm{Fe}$ phonon partial DOS in $\mathrm{Fe}_{3} \mathrm{Al}$ depends much more strongly on SRO than on LRO. This is encouraging

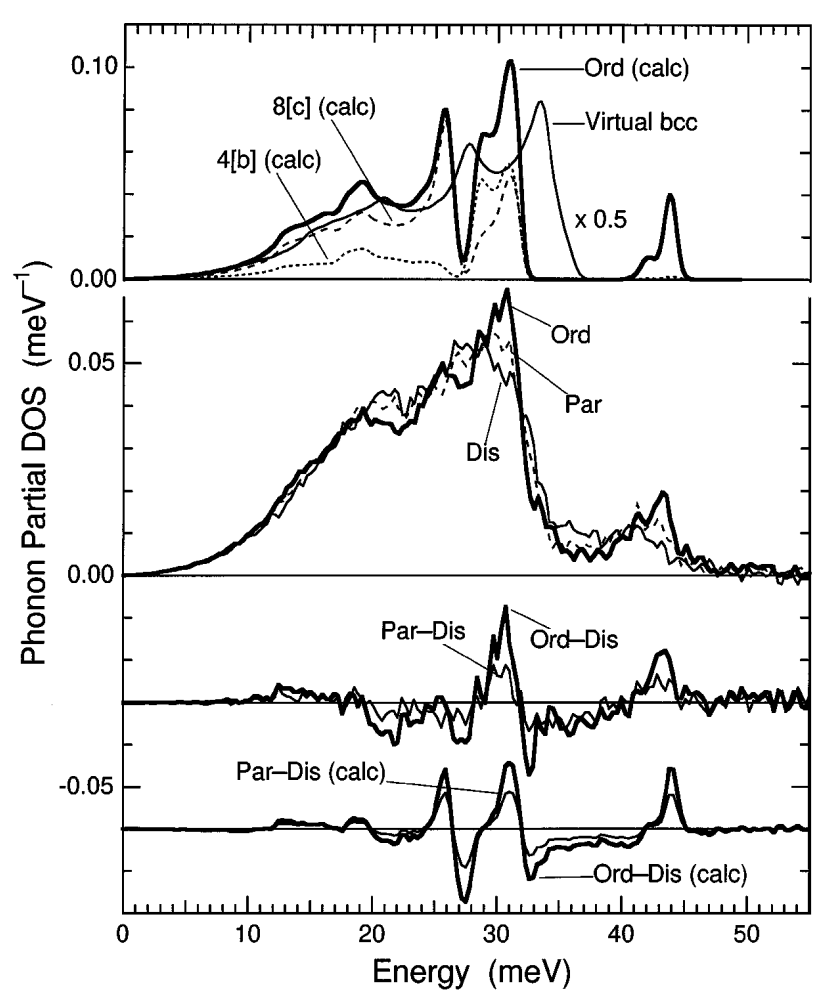

FIG. 4. Phonon partial DOS curves for ${ }^{57} \mathrm{Fe}$ in ${ }^{57} \mathrm{Fe}_{3} \mathrm{Al}$. Top: Calculated curves using interatomic force constants for the alloys with disorder and $\mathrm{DO}_{3}$ chemical order [7]. Upper middle: Experimental phonon partial DOS curves obtained from the spectra of Fig. 3. Lower middle: Differences of experimental phonon partial DOS curves. Bottom: Calculated difference of phonon partial DOS curves, using the local approximation described in text.

for the use of the vibrational effective cluster interaction method [6], for example.

We have made a quantitative test of a local cluster approximation which assumes the phonon partial DOS for a ${ }^{57} \mathrm{Fe}$ atom depends only on the number $n$ of its $1 \mathrm{nn}$ $\mathrm{Al}$ atoms. We obtained these numbers $n$ from the hyperfine magnetic field distributions shown in Fig. 2(b) by fitting them to Gaussian functions. The three samples had the following fractions of $\{0,1,2,3,4,5\} 1 \mathrm{nn} \mathrm{Al}$ atoms: disordered $\{0.10,0.21,0.30,0.21,0.15,0.02\}$, partially ordered $\{0.18,0.14,0.17,0.22,0.26,0.02\}$, and ordered $\{0.16,0.20,0.10,0.19,0.33,0.01\}$. To help with the assignment of phonon partial DOS curves to specific chemical environments, we used a Monte Carlo simulation of ordering on a bcc lattice, described previously [14], to generate $A_{3} B$ alloys with $5.24 \times 10^{5}$ atoms and various states of chemical order. The $A$ atoms (denoted $\mathrm{Fe}$ ) having the numbers $n$ of first neighbor $B$ atoms (denoted $\mathrm{Al}$ ) were counted, and an alloy was obtained with the following fractions of $\{0,1,2,3,4,5\} 1 \mathrm{nn} B$ atoms: $\{0.21,0.12,0.083,0.18,0.40,0.004\}$. This was similar to our ${ }^{57} \mathrm{Fe}_{3} \mathrm{Al}$ ordered alloy, especially considering that the zero and one Al neighborhoods cannot be reliably resolved experimentally. The purpose of this simulation 
was not to match the ordered alloy in detail, however, but rather to obtain the spatial autocorrelation functions for $\mathrm{Fe}$ atoms having the various $1 \mathrm{nn} \mathrm{Al}$ atom neighborhoods. This was done by taking the Fourier transform of the spatial arrangement of $\mathrm{Fe}$ atoms having each number $n$ of Al neighbors, $\Psi_{n}(\mathbf{k})$, and calculating the "diffracted intensity," $I_{n}(\mathbf{k})=\Psi_{n}^{*}(\mathbf{k}) \Psi_{n}(\mathbf{k})$.

As expected, these diffracted intensities peaked at values of $\mathbf{k}$ that were reciprocal lattice points of the bcc lattice, $\mathbf{g}$. The integrated intensities for the six $I_{n}(\mathbf{g})(0 \leq$ $n \leq 5$ ) were confirmed to be accurately in the ratio of the number of $\mathrm{Fe}$ atoms having $n \mathrm{Al}$ atoms as first neighbors. Intensities in the superlattice diffractions at $\left(\frac{1}{2} \frac{1}{2} \frac{1}{2}\right)$ and (100) reciprocal lattice points are expected when $\mathrm{DO}_{3}$ chemical order is present. For the ordered alloy in the simulation, the integrated intensity of $I_{0}\left(\frac{1}{2} \frac{1}{2} \frac{1}{2}\right)$ was nearly one (0.86), the intensity of $I_{4}\left(\frac{1}{2} \frac{1}{2} \frac{1}{2}\right)$ was nearly zero $(0.00)$, and the intensity of $I_{4}(100)$ was nearly one (0.99). These results show that the $\mathrm{Fe}$ atoms with zero and four $1 \mathrm{nn} \mathrm{Al}$ atoms have spatial arrangements consistent with the $\mathrm{DO}_{3}$-ordered structure, as expected.

We assigned the $8[\mathrm{c}]$ and $4[\mathrm{~b}]$ phonon partial DOS curves to the fractions of $\mathrm{Fe}$ atoms with $n \mathrm{Al}$ neighbors, depending on the relative intensities of their $I_{n}\left(\begin{array}{lll}\frac{1}{2} & \frac{1}{2} & \frac{1}{2}\end{array}\right)$ and $I_{n}(100)$ diffractions in the simulated alloy. From the square roots of the intensities of the superlattice diffractions, we defined parameters for $\mathrm{DO}_{3}$ and $\mathrm{B} 2$ chemical order in a conventional way [14]. When the largest of these order parameters was less than one, the remainder was attributed to a fraction of bcc periodicity for the $\mathrm{Fe}$ atom. We then assigned the calculated $4[\mathrm{~b}]$ partial DOS to the $\mathrm{DO}_{3}$ chemical order parameter, the calculated $8[\mathrm{c}]$ partial DOS to the $B 2$ chemical order parameter, and the experimental phonon partial DOS from the disordered alloy to the residual bcc parameter. (Approximately, the $4[\mathrm{~b}]$ partial DOS was assigned to $\mathrm{Fe}$ atoms having zero and one $\mathrm{Al}$ neighbors, the disordered DOS was assigned to $\mathrm{Fe}$ atoms having two $\mathrm{Al}$ neighbors, and the $8[\mathrm{c}]$ partial DOS was assigned to $\mathrm{Fe}$ atoms having three, four, or five $\mathrm{Al}$ neighbors.)

The calculated difference in the phonon partial DOS of the ordered and disordered alloys is shown as the thick solid curve at the bottom of Fig. 4. The experimental difference is shown above it in a comparison that involves no scaling or adjustable parameters. We are surprised by the excellent overall agreement between the calculated and experimental results in both overall magnitude and in individual features. The only discrepancy is around $26 \mathrm{meV}$, but this discrepancy is found for the calculated partial DOS for the ordered alloy itself.

A general relationship between the phonon DOS and chemical short-range order may be expected from the slopes of phonon dispersion curves. Flat dispersion curves provide a high density of phonon states, especially when they include Brillouin zone boundaries. They also provide a slow group velocity of sound. Slow propagation of a wave packet of energetic phonons requires that much of the energy of lattice vibrations is associated with localized atom movements. We expect localized atom movements to be strongly affected by SRO. We should expect SRO to have a major effect on the phonon DOS because of the high density of phonon states associated with these localized atom movements. Those parts of the phonon DOS not associated with flattened dispersion curves should be less sensitive to chemical SRO. It is interesting that for energies below $12 \mathrm{meV}$, where the dispersion curves have a significant slope [10], the local environment model predicts the wrong sign for the difference in the partial DOS. (The measured difference is rather small in this low energy range, however.) We note that this interpretation is at odds with interpretations of vibrational entropies of order-disorder transformations based on a Debye model with a constant velocity of sound.

The work at Caltech was supported by the U.S. National Science Foundation under Contract No. DMR9415331, and the work at Argonne was supported by the U.S. Department of Energy under Contract No. W-31109-ENG-38.

[1] F. Ducastelle, Order and Phase Stability in Alloys (NorthHolland, Amsterdam, 1991).

[2] J. K. Okamoto et al., Microbeam Analysis-1990, edited by J. R. Michael and P. Ingram (San Francisco Press, San Francisco, 1990), p. 56.

[3] A. F. Guillermet and G. Grimvall, J. Phys. Chem. Solids 53, 105 (1992).

[4] L. Anthony et al., Phys. Rev. Lett. 70, 1128 (1993).

[5] S. J. Clark and G. J. Ackland, Phys. Rev. B 48, 10899 (1993).

[6] G. D. Garbulsky and G. Ceder, Phys. Rev. B 49, 6327 (1994); 53, 8993 (1996).

[7] L. Anthony et al., Phys. Rev. Lett. 73, 3034 (1994).

[8] B. Fultz et al., Phys. Rev. B 52, 3315 (1995).

[9] C. Van Dijk, Phys. Lett. 34A, 255 (1970).

[10] I. M. Robertson, J. Phys. Condens. Matter 3, 8181 (1991).

[11] W. Sturhahn et al., Phys. Rev. Lett. 74, 3832 (1995).

[12] M. Seto et al., Phys. Rev. Lett. 74, 3828 (1995).

[13] T. S. Toellner et al., Appl. Phys. Lett. 71, 2112 (1997).

[14] Z. Q. Gao and B. Fultz, Philos. Mag. B 67, 787 (1993).

[15] G. Le Caër and J. M. Dubois, J. Phys. E 12, 1083 (1979).

[16] W. Marshall and S.W. Lovesey, Theory of Thermal Neutron Scattering (Oxford University, London, 1971), p. 94.

[17] V.F. Sears, Phys. Rev. A 7, 340 (1973).

[18] L. J. Nagel et al., Philos. Mag. B 5, 681 (1997). 